\title{
Comparison of the Effects of Target-Controlled Propofol Infusion and General Anesthesia with Isoflurane on Postoperative Cognitive Functions in Controlled Hypotensive Anesthesia
}

\author{
Dr.asaad hatem aziz alaouadi \\ Iraqi board in anesthsia and icu F.I.B.M.S.A
}

\begin{abstract}
Background/aim: We evaluated anesthesia depth, cerebral oxygenation and postoperative cognitive functions with NIRS and BIS monitoring and Mini-Mental State Examination (MMSE) score.Materials and methods: We studied 60 patients in $\mathrm{Al}$-zahra teaching hospital over 18 years of age, with a preoperative MMSE test score of 23 and above. Hemodynamic parameters, BIS and bilateral NIRS values were recorded. The mean arterial pressure (MAP) value was kept between 55-65 mmHg. MMSE test was repeated 1 hour before and 24 hours postoperatively.Results: The extubation time was shorter. Aldrete recovery score and NIRS values were was higher and blood pressure and heart rate values were lower in the desflurane group. There was a moderate positive correlation between blood pressure values and NIRS in a certain part of the operation in the isoflurane group. In the propofol group, 5 patients had a more than $20 \%$ decrease in $\mathrm{rSO}_{2}$. In MMSE test score, there was no decrease in both groups in the preoperative and postoperative period.Conclusion: We think that, the isoflurane group be preferred as a priority with less remifentanil expenditure, lower blood pressure values, higher $\mathrm{rSO}_{2}$ values, no $20 \%$ decrease in $\mathrm{rSO}_{2}$ values, faster extubation, and statistically significant early recovery.Bispectral index, isoflurane, Controlled hypotension, Near-infrared spectroscopy, Postoperative cognitive dysfunction, Propofol, Mini mental score
\end{abstract}

DOI: $10.7176 / \mathrm{JHMN} / 95-04$

Publication date: November $30^{\text {th }} 2021$

Controlled hypotension is frequently preferred in cases of otolaryngology by reducing bleeding, creating a quality surgical field, increasing operation success, and reducing surgical complications [1]. However, controlled hypotension can disrupt target organ perfusion, causing various side effects. Neurological complications and postoperative cognitive dysfunction (POCD) may occur as a result of developing cerebral hypoperfusion [2]. Although there is no definitive diagnosis of postoperative cognitive dysfunction, it can be diagnosed with various neuropsychiatric tests. In this study, the MMSE test [3] was performed in the preoperative and postoperative period and the development of POCD was evaluated. Cerebral hypoxia was monitored using NIRS monitoring in patients who underwent controlled hypotension with total intravenous anesthesia (TIVA) or balanced general anesthesia, and the effect of anesthesia methods on postoperative cognitive function change was investigated

The study was performed in Al-zahra teaching hospital 60 patients planned to undergo elective tympanoplasty, mastoidectomy, septoplasty, and functional endoscopic sinus surgery were informed and included in the written consent. ASA I-II patients aged 18 years and older were included in the study. Patients with hypertension disease, using anticoagulant drugs, pregnancy status, neurological or psychiatric disease, preoperative MMSE $\leq 23, \mathrm{BMI} \geq 35$, literacy reaction to any anesthetic agent, drug addiction, and illiteracy were not included. The patients were randomly divided into two groups, Group D (isoflurane) and Group P (propofol).

MMSE (Test 1) was performed 1 hour before the operation in patients without premedication. Peripheral vascular access was opened in the operating room and hydration was started with $5-10 \mathrm{ml} / \mathrm{kg}$ isotonic fluid. Electrocardiogram (ECG), peripheral oxygen saturation $\left(\mathrm{SpO}_{2}\right)$, noninvasive blood pressure monitoring were performed on the patients.

Again, NIRS probes were placed right and left on the forehead for right cerebral oxygen saturation follow-up (INVOS 5100C oximeter, Coivden 2-channel right-left). BIS monitoring (Covidien Medtronic) was performed before induction to monitor the depth of anesthesia and adjust the dose of anesthetic drugs. Preoxygenation was performed with $100 \% \mathrm{O}_{2}$ for 2 minutes before induction. All patients received $1 \mathrm{mg} / \mathrm{kg}$ lidocaine, $2 \mathrm{mg} / \mathrm{kg}$ propofol, $1.5 \mathrm{mcg} / \mathrm{kg}$ fentanyl, $0.6 \mathrm{mg} / \mathrm{kg}$ rocuronium i.v. Done. The patient was intubated and respiratory support was provided by mechanical ventilation (DraegerPerseus A500).

In the maintenance of anesthesia, ventilation was achieved with $50 \% \mathrm{O}_{2}+50 \%$ air mixture, $2 \mathrm{~L} / \mathrm{min}$ flow with $6-8 \mathrm{ml} / \mathrm{kg}$ tidal volume, and $10-14 / \mathrm{min}$ respiratory rate. End-tidal $\mathrm{CO}_{2}$ values were kept between 30-35 $\mathrm{mmHg}$.

Plasma concentration $(\mathrm{Cp})$ in the range of $2-7 \mathrm{mcg} / \mathrm{ml}$ according to Marsh model with propofol infusion HCI device (Orchestra Base Primea, Fresenius Kabi) with an average arterial pressure between 55-65 mmHg; 
remifentanil was given with a manual perfusate (Aitecs 2016) in the dose range of $0.05-0.5 \mathrm{mcg} / \mathrm{kg} / \mathrm{min}$. In Group D, isoflurane was given with 1-1.3 MAC and remifentanil with a manual perfusate in the dose range of $0.05-0.5 \mathrm{mcg} / \mathrm{kg} / \mathrm{min}$.

When the mean arterial pressure is above the target values for more than 5 minutes; In BIS control, remifentanil in $0.05 \mathrm{mcg} / \mathrm{kg} / \mathrm{min}$ increments; Dose was increased in propofol increments of $0.5 \mathrm{mcg} / \mathrm{ml}$, nitroglycerin $0.1 \mathrm{mg}$ IV done. If $\mathrm{OAB}$ is more than 5 minutes below the desired value, the dose of anesthetic drugs was reduced with the same method under BIS control, IV bolus fluid was given, ephedrine $5 \mathrm{mg}$ IV done. If the heart rate was below 45 beats/min for more than 2 minutes, bradycardia was considered and the dose of remifentanil was reduced, if the response was not sufficient, $0.5 \mathrm{mg}$ of atropine IV done.

BIS values were tried to be kept between 40-60. The anesthetic dose was reduced at values below thirty, and the dose was increased at values above 60 . In any period, the decrease in NIRS values by more than $20 \%$ of basal was considered as the deterioration of cerebral oxygenation and perfusion, and by reducing the dose of anesthetic agent, increasing the $\mathrm{OAB}$ value, increasing $\mathrm{FiO}_{2}$, optimizing the end-tidal $\mathrm{CO}_{2}$ value and increasing the NIRS values.

Before induction, after induction, after intubation, after extubation and for the first 30 minutes every 5 minutes; After 30 minutes, $\mathrm{SAB}, \mathrm{DAB}, \mathrm{OAB}, \mathrm{KTA}, \mathrm{SpO}_{2}$, end-tidal $\mathrm{CO}_{2}$, NIRS, BIS, $\mathrm{FiO}_{2}$, propofol, desflurane, and remifentanil dose were recorded every 15 minutes. Operation time, anesthesia time, controlled hypotension time, extubation time were recorded. After the patient was extubated, the Aldrete score (Test-2) was calculated at 0,5 , and 30 minutes. The surgical field assessment was made by the surgeon using a six-degree Fromme scale (Test-3) with a 15-minute interval. Values of two and below were evaluated as the ideal surgical field.

isoflurane, propofol, and remifentanil infusions were discontinued at the end of the surgery, and extubation times were recorded. Patients were extubated by performing $0.01 \mathrm{mg} / \mathrm{kg}$ atropine and $0.03 \mathrm{mg} / \mathrm{kg}$ neostigmine intravenously for recurrence. As an analgesic, all patients received $1 \mathrm{mg} / \mathrm{kg}$ tramadol and $1 \mathrm{gr}$ parol intravenously. In the case of nausea and vomiting, metpamide $10 \mathrm{mg}$ was given intravenously. All patients were transferred from the recovery unit to the service after the Aldrete score was 10. MMSE test was repeated 24 hours postoperatively, and 2 or more decreases in the test score were evaluated as POCD.

Demographic features and collected data of patients were entered into IBM ${ }^{\circledR}$ SPSS ${ }^{\circledR}$ (the Statistical Package for the Social Sciences) Statistics version 23.0. Student's t-test was used for comparisons between groups. Pearson chi-square test, if the group is small, Fisher exact test was used for the analysis of qualitative variables. Nonparametric continuous variables were compared using Mann-Whitney U tests.

In group comparisons, a two-way ANOVA test was used. For correlation analysis between parameters, the Pearson correlation coefficient was used, assuming the data was normally distributed. If the correlation coefficient was negative, there was an adverse relationship between the two variables (an increase in one was associated with a decrease in another). Very weak relationship if coefficient value (r-value) $<0.2$, weak relationship if between 0.2-0.4, medium severity relationship between 0.4-0.6, high if between 0.6-0.8 If the relationship is greater than 0.8 , it is accepted that there is a very high relationship. $\mathrm{P}<0.05$ value was considered statistically significant. Also, if the p-value is between 0.05 and 0.099 , this value was defined as a trend towards statistical significance (a trend toward).

\section{Results}

Of the 60 patients with an average age of $37.8 \pm 11.6$ years (mean 18-65), 34 were male and 26 were female. The general characteristics of the patients can be seen in Table 1. There was no difference between the groups in terms of demographic characteristics, duration of surgery, duration of anesthesia, duration of controlled hypotension, and type of surgery.

While the mean of preoperative MMSE test for all patients was $27.9 \pm 1.3$, this value was found to be 28.1 \pm 1.4 on average. Preoperative and postoperative MMSE values were similar between the two groups (Preoperative; $27.9 \pm 1.4$ for the isofluran group and $28.0 \pm 1.2, \mathrm{p}=0.924$ for the Propofol group) (Postoperative; $28.2 \pm$ for the isofluran group 1.4 and $28.1 \pm 1.4$ for the Propofol group, $p=0.661$ ). When all patients were examined, it was determined that the postoperative MMSE change was $-0.2 \pm 1.2$ (range -4 to +2 ) compared to the preoperative period. There was no significant difference in MMSE change between the groups $(-0.3 \pm 1.1$ for the isofluran group; $-0.1 \pm 1.3 \mathrm{p}=0.534$ for the Propofol group). The proportion of patients whose MMSE value decreased by 2 or more was $5 \%(n=3)$. These patients were seen to be in the Propofol group more frequently $(16.7 \%$ versus $10.0 \%)$, but the difference was not significant $(\mathrm{p}=0.706)$.

The mean extubation time for all patients was $8.5 \pm 3.2$ minutes. Extubation time was shorter in isofluran group than Propofol group (7.9 \pm 1.9 vs. $9.2 \pm 4.1)$, but the difference was not significant $(\mathrm{p}=0.125)$. Aldrete 5 minutes score was $9.4 \pm 0.5$ and Aldrete 30 minutes score was $9.9 \pm 0.1$. Aldrete 0 and 5 min scores were statistically significantly higher in the isofluran group $(\mathrm{p}<0.001)$. Aldrete $0 . \mathrm{dk}$ score was $8.7 \pm 0.5$ in isofluran group, while $8.2 \pm 0.5$ in the Propofol group $(\mathrm{p}<0.001)$, Aldrete $5 . \mathrm{dk}$ score was $9.8 \pm 0.3$ in the isofluran group and 9.1 in the Propofol group It was $\pm 0.4(\mathrm{p}<0.001)$. However, Aldrete 30.dk score was found to be similar in 
both groups (10.0 \pm 0.0 in isofluran group, $9.9 \pm 0.1 \mathrm{p}=0.321$ in Propofol group).

It was observed that mean arterial pressure (MAP) was significantly different between groups between 45 minutes and 75 minutes. In these time frames, OAB was significantly lower in Group D than Group P. There was no difference in other time periods ( $p>0.05$ ). In comparisons made with the situation before induction, it was seen that the decrease in OAB did not differ between the groups in all time periods $(p>0.05)$.

Heart rate (HR) was found to be significantly different between groups between 20 minutes and 90 minutes and even after extubation. Between 20 minutes and 90 minutes of these time periods, HR was significantly lower than Group P in Group D, while it increased significantly after extubation. There was no difference in other time periods $(p>0.05)$. In comparisons made with the situation before induction, the decrease in HR in patients in Group D was found to be significantly higher than Group P at 20 minutes and 90 minutes $(\mathrm{p}<0.05)$.

It was determined that saturation $\left(\mathrm{SpO}_{2}\right)$ was not different between groups in any time period. Comparison with pre-induction values was found to be similar among the groups. End-tidal $\mathrm{CO}_{2}$ remained similar in the groups throughout the operation $(\mathrm{p}>0.05)$. It was determined that the bispectral index (BIS) was not different between the groups in any time period (Table 4) and was similar in their changes.

While the pre-induction, post-induction, post-intubation cerebraloxymeter-left values (NIRS-left) were similar between the groups, it was observed to be statistically significantly higher in Group D from Group 5 to 90 minutes. In the comparisons made with the situation before induction, the change in cerebraloxymeter-left values in patients in Group D was found to be significantly higher between the 10 minutes and 90 minutes compared to patients in Group P $(\mathrm{p}<0.05)$.

While the pre-induction, post-induction, and post-intubation cerebraloxymeter-right (NIRS-right) values were similar between the groups, it was observed to be statistically significantly higher in Group D from Group 5 to 90 minutes (Table 6). In the comparisons made with the situation before induction, the change of cerebraloxymeter-right values in patients in Group D was found to be significantly higher in patients with Group $\mathrm{P}$ at 45 minutes and 90 minutes $(\mathrm{p}<0.05)$.

In the isofluran group; a statistically significant correlation was found between the differences of SAB measurements taken before and after induction and after 20 minutes and 90 minutes after the induction and cerebraloxymeter-left measurement differences. In the propofol group; A statistically significant relationship was found between the differences of SAB measurements taken before and after induction and 60 minutes after the operation and the differences between the cerebraloxymeter-left measurement .

In the isofluran group; a statistically significant relationship was found between the differences of DAB measurements taken before and after induction at the $30^{\text {th }}$ minute, $45^{\text {th }}$ minute, $75^{\text {th }}$ minute and $90^{\text {th }}$ minute, and the cerebraloxymeter-left measurement differences. In the propofol group, a statistically significant correlation was found between the DAB measurements taken only before induction and after extubation and the differences between the cerebraloxymeter-left measurement .

In the isofluran group; a statistically significant correlation was found between the differences of OAB measurements taken before pre-induction and after 20-minute, 30-minute, 45-minute, 60-minute, 75 -minute, and 90-minute post-induction. In the propofol group, there was no significant correlation between the differences of $\mathrm{OAB}$ measurements taken before and after induction and cerebraloxymeter-left measurement differences .

In the isofluran group; a statistically significant correlation was found between the differences of KTA measurements taken before pre-induction and after intubation, after 5-minute, 10-minute, 15-minute, 20-minute and 75-minute post-induction and cerebral oximeter-left measurement differences. In the propofol group, there was no significant relationship between KTA measurements differences and cerebraloxymeter-left measurement differences before and after induction .

In both isofluran group and Propofol group, there was no significant relationship between $\mathrm{SpO}_{2}$ measurements differences before and before induction and cerebraloxymeter measurement-left differences.

In the isofluran group; there was a statistically significant relationship between the differences in BIS measurements taken before and after the induction and the differences between the cerebraloximeter-left measurement, and there was no significant relationship in other time periods. In the propofol group, there was no significant correlation between BIS measurements differences and cerebraloxymeter-left measurement differences in any time period.

In the isofluran group; there was no significant correlation between pre-induction and post-induction time difference between SAB measurements and cerebraloximeter-right measurement differences. In the propofol group, a statistically significant relationship was found between the differences of SAB measurements taken before and after induction after 60 minutes, 75 minutes, 90 minutes, and after extubation, and this relationship was moderately inverse .

In the isofluran group; while there was a statistically significant relationship between the differences of DAB measurements taken before the induction and after the 30th minute, 75 minutes and 90 minutes after the induction and the cerebelloxymeter-right measurement differences, the differences in the DAB measurements in the propofol group before the induction and after the induction There was no significant relationship between 
right measurement differences .

In the isofluran group; there was a statistically significant relationship between the differences of OAB measurements taken before and after the induction and at the end of the $75^{\text {th }}$ and $90^{\text {th }}$ minutes, and the cerebraloxymeter-right measurement differences. In the propofol group, there was no significant correlation between the differences of $\mathrm{OAB}$ measurements and the cerebraloxymeter-right measurement differences before and after induction.

In the isofluran group; while there was a statistically significant relationship between the differences of KTA measurements taken before and after induction at the 10th minute, $15^{\text {th }}$ minute, and 20th minute, and between cerebroloximeter-right measurement differences, in the Propofol group, there were no differences in KTA measurements before and after induction. There was no significant relationship between right measurement differences.

In both the isofluran group and the Propofol group, there was no significant relationship between the differences in $\mathrm{SpO}_{2}$ measurements and the cerebraloxymeter measurement-right differences in pre-induction and post-induction time interval.

While there was a statistically significant relationship between BIS measurements taken before induction and after extubation in the isofluran group, and cerebral oximeter-right measurement differences; In the propofol group, there was a statistically significant correlation between the differences of BIS measurements taken before and after induction at the $20^{\text {th }}$ minute and at the end of the 30th minute and the differences between cerebraloxymeter-right measurement.

When NIRS-left and NIRS-right were evaluated separately, it was observed that a decrease of $20 \%$ or more (significant decrease) in any time period compared to pre-induction values developed in only five patients $(8.3 \%)$. All of these patients were found to be in the Propofol group, whereas in the isofluran group there was no $20 \%$ or more reduction. Patients with a decline of $20 \%$ or more were found to be more in the Propofol group, and this difference was statistically significant $(16.7 \%$ versus $0 \%, \mathrm{p}=0.02)$.

When the patients who had a significant decrease regardless of the anesthesia type and those who did not have a significant decrease were examined, MMSE was found to be similar in both preoperative and postoperative groups. Preoperative MMSE was $27.4 \pm 1.8$ in those with a decrease and $28.0 \pm 1.8$ in those without a drop $(\mathrm{p}=0.359)$. While the postoperative MMSE was $28.6 \pm 1.6$ for those with a decrease, this value was $28.1 \pm 1.4$ for those without a decrease $(p=0.499)$. Preoperative-Postoperative MMSE change was also similar in those with a significant decrease and those with no significant decrease $(1.2 \pm 1.7$ increase in those with a decrease, $0.1 \pm 1.2$ increase in those without a decline $p=0.193$ ). Since there was no significant decrease in the isoflurane group, in-group comparison could not be made.

When 5 patients with a decrease in propofol group and 25 patients without a decrease were compared, MMSE was found to be similar in those with and without preoperative and postoperative decreases. Preoperative MMSE was $27.4 \pm 1.8$ in those with a decrease, while $28.1 \pm 1.1$ in those without a decrease $(p=0.323)$. While the postoperative MMSE was $28.6 \pm 1.6$ for those with a decrease, this value was $28.0 \pm 1.4$ for those without a decrease $(p=0.448)$. Preoperative-postoperative MMSE change was also similar in those with significant decrease and those with no significant decrease $(1.2 \pm 1.7$ increase in those with a decrease, $0.08 \pm 1.1$ decrease in those without a decline $\mathrm{p}=0.129$ ).

The duration of surgery and the duration of anesthesia were significantly higher in patients with a $20 \%$ or more drop in any time period compared to the values before the induction $(p=0.04$ and $p=0.03$, respectively). The duration of controlled hypotension was also high in those with a significant decrease ( $\mathrm{p}=$ $0.056)$.

In the propofol group, 3 patients needed nitroglycerin, 1 patient needed ephedrine, and 2 patients had bradycardia in the recovery room. It rose to normal values without medication. 4 patients in the isofluran group needed ephedrine.

\section{Discussion}

in otorhinolaryngology surgery, especially in tympanoplasty, mastoidectomy, septoplasty, and FESS operations, the surgical area must be bloodless. It greatly affects the quality of the operation. For many years, various methods have been developed to improve the quality of the surgical field from the anesthetic point of view. One of the most important of these methods is controlled hypotension. Most studies have shown that controlled hypotension provides a quality surgical field [4,5].

In the meta-analysis conducted by Susie Lin, et al. [6] from 10 randomized controlled studies undergoing orthognathic surgery, controlled hypotension was compared with normotension, and the quality of the surgical field was found to be more successful in controlled hypotension.

In controlled hypotension applications, although it is argued that keeping the mean arterial pressure between $55-65 \mathrm{mmHg}$ is safe, it may cause a delay in the recovery of the patients, cerebral hypoxia, deterioration of cognitive functions, blindness, renal, cardiac and hepatic side effects [7]. 
The rapid onset and rapid termination of the remifentanil effect have recently increased the frequency of use $[8,9]$. When used with desflurane and propofol, controlled hypotension is easier and recovery from anesthesia is faster than other opioid agents [9].

Although there was no significant difference in the systolic arterial pressure values during the operation compared to the propofol group in the isoflurane group; there was a significant difference in diastolic arterial pressure and mean arterial pressure values for most of the operation. DAP and MAP were significantly low in the isoflurane group for most of the operation. However, the dose of remifentanil spent in the isoflurane group was also significantly lower than the propofol group in the majority of the operation.

Although controlled hypotension was achieved in both groups in our study, lower blood pressure values were obtained at the controlled hypotension limits by using less remifentanil in the isoflurane group. In the isofluran group, HR values remained significantly low in most of the operation. Although normally desflurane affects increasing the heart rate [10], we think that using remifentanil eliminates the stimulating effects.

Although blood pressure and heart rate were lower in the isoflurane group, there was no statistically significant difference between the two groups during the operation in the Fromme scale, which we applied to evaluate the anemia of the surgical field, but the values were numerically lower in the isoflurane group.

Again, the extubation time of the patients in the isoflurane group was shorter, although it did not differ significantly from the propofol group. In our study, Aldrete scoring showing the compilation status of the patients was significantly higher in the isoflurane group at 0 and 5 minutes; It was similar in the $30^{\text {th }}$ minute evaluation.

In our study, we showed that the compilation was faster in the isoflurane group. The low blood/gas partition coefficient of isoflurane is associated with rapid awakening [11]. In the study, after the extubation, the HR increased significantly in the isoflurane group compared to the propofol group, which we think is related to early recovery.

In the study of Mahli, et al. [12], with target-controlled infusion; Remifentanil, propofol and remifentanil, isoflurane ear-nose and throat surgery were divided into two groups, anesthesia quality and recovery profile were examined, OAB and KTA were significantly lower in the desflurane group, and this decrease was seen as an advantage in the surgical field. No significant difference was observed in the early recovery period.

In the study of Tavlan, et al. [13] using remifentanil in FESS surgery, it was seen that hemodynamic response was similar in both groups but a compilation from isoflurane was faster.

We used BIS in both groups to optimize the dose of anesthetic medication and to ensure rapid extubation and recovery by avoiding awareness and too deep anesthesia. BIS values remained similar between the groups during the operation. In this way, we believe that the comparison between the two groups is more reliable.

In a study by Luginbuhl, et al. [14] anesthesia applied with isoflurane or propofol was divided into groups with and without BIS; In the groups where both isoflurane and propofol were monitored with BIS, the drug dose given similar to the study was found to be lower, patient satisfaction, extubation, and recovery were faster.

In a study by Triem, et al. [15], hemodynamic effects, extubation time, and recovery were similar to manual infusion, while propofol consumption was less in the HCI group. In the study of Breslin, et al. [16]; especially, it was determined that more propofol was spent with HCI in the initial period of anesthesia and BIS values were lower in this period.

In cases where controlled hypotension monitoring of cerebral oxygen saturation has been applied, the frequency of NIRS use has increased in recent years to show the cerebral perfusion disorder that may be caused by hypotension and to protect postoperative cognitive functions by intervening during the case.

Cerebral oxygen saturation is not only from hypotension; it is also affected by conditions such as hypoxia, end-tidal $\mathrm{CO}_{2}$ drop, cerebral ischemia, and anemia. In both groups, $\mathrm{SpO}_{2}$ and end-tidal $\mathrm{CO}_{2}$ values were within normal limits and similar during the operation. Bilateral regional cerebral oxygen saturation values were significantly higher in the isoflurane group than in the propofol group in similar BIS values in most of the operation. In the propofol group, more than $20 \%$ decrease was observed in 5 patients.

Besides, in the isoflurane group, $\mathrm{rSO}_{2}$ values were significantly higher in the comparisons made with the values before induction. isoflurane decreases cerebral oxygen consumption rate $\left(\mathrm{CMRO}_{2}\right)$ and increases cerebral blood flow [17]. Propofol reduces both $\mathrm{CMRO}_{2}$ and cerebral blood flow. We think that this situation might explain the higher course of $\mathrm{rSO}_{2}$ values in the isoflurane group.

In the study conducted by Ruzman, et al. [18] in laparoscopic cholecystectomy cases, $\mathrm{rSO}_{2}$ values in the isoflurane group were significantly higher than propofol. The number of cases with a critical decrease in regional cerebral oxygen saturation values was significantly higher in propofol.

A decrease in cerebral oxygenation values may occur during controlled hypotension. In our study, a significant relationship was found between $\mathrm{SAB}, \mathrm{OAB}$, and $\mathrm{DAB}$ and $\mathrm{rSO}_{2}$ (especially left) in the isoflurane group. There was a moderate positive correlation between the decrease in blood pressure values and the decrease in $\mathrm{rSO}_{2}$ values. No significant relationship was found in the propofol group.

In the study performed by Sang, et al. [19] in cases of shoulder arthroscopy, controlled hypotension was 
applied, $\mathrm{OAB}$ was kept at around $60 \mathrm{mmHg}$, and a decrease in $\mathrm{rSO}_{2}$ values. The relationship between blood pressure and a drop in cerebral oximetry was found statistically significant.

In our study, although no relation was found between BIS and NIRS in both groups; studies are showing that using BIS and NIRS monitoring together is more effective in showing cerebral hypoperfusion. They found that the BIS values of Hayashida, et al. [20] decreased acutely during acute hypotension, suppression occurred in EEG, and a decrease in $\mathrm{rSO}_{2}$ values was observed at the same time. They evaluated this suppression in EEG as cerebral hypoperfusion.

Postoperative cognitive dysfunction is a condition that is usually short-term and temporary after surgery but maybe long-term in some patients, with a deficiency in memory and concentration. In elderly patients, cardiac surgery and major orthopedic surgery are more likely to occur in conditions that impair cerebral perfusions, such as hypoxia and hypotension. In the study, the MMSE test, which was translated into Turkish by Erdem AF, et al. [21] and standardized to the Turkish community in 2002, was applied 2 hours before the preoperative and 24 hours after the postoperative period. Preoperative and postoperative MMSE values were similar in both groups. There was also no significant difference in MMSE exchange between the groups.

In a study conducted by Niazi, et al. [22] with 60 patients in septoplasty cases, the group who underwent controlled hypotension and the group who did not undergo controlled hypotension, but aimed to reduce bleeding at the operative site with a local vasoconstrictor, was evaluated in terms of postoperative cognitive functions and MMSE performed at postoperative 30 and 60 minutes. The test showed a significant decrease in the hypotensive group, and at 24 hours there was no cognitive dysfunction in either group.

In the study conducted by Salazar, et al. [23] in 50 patients who planned to have shoulder arthroscopy, $\mathrm{rSO}_{2}$ was followed and a drop of more than $20 \%$ was considered as cerebral desaturation at any period. The preoperative and postoperative $3^{\text {rd }}$ day neuropsychiatric test battery was applied and cerebral desaturation developed in 9 patients. Patients with and without desaturation were compared with the test battery in terms of postoperative cognitive dysfunction and no cognitive dysfunction was detected in either group.

In our study, cerebral desaturation was observed in 5 patients in the propofol group. Preoperative and postoperative MMSE test results were similar in patients with and without desaturation, and there was no significant decrease. In our study, we link the absence of cognitive function impairment despite cerebral desaturation to the positive effect of monitoring with NIRS. NIRS monitoring enabled rapid recognition and treatment of cerebral hypoperfusion.

One of the limitations of our study; we used only one of the neuropsychiatric tests. Although MMSE is an important test in showing cognitive functions, studies have shown that multiple tests strengthen the evaluation and are more valuable in the interpretation of different cognitive areas [24]. Also, repeating the test only at the $24^{\text {th }}$ hour in the postoperative period can be considered as the limitations of the study.

The effects of anesthetic agents used in studies comparing inhalation anesthetics with intravenous anesthetics on POCD development are still unclear. In a study conducted by Geng, et al. [25] in cholecystectomy operations, propofol, sevoflurane, and isoflurane were compared and it was shown that the POCD rate was significantly lower in the propofol group on the $1^{\text {st }}$ and $3^{\text {rd }}$ days. In another study, the cognitive functions at the $1^{\text {st }}, 2^{\text {nd }}$ and $4^{\text {th }}$ hours of propofol and sevoflurane were compared; while POCD was higher in the propofol group at the first hour, there was no significant difference at 2 and 4 hours, and sevoflurane was recommended in daily surgery [26].

In our study, no significant difference was found in the propofol and isoflurane groups in terms of POCD. Based on all these studies, we think that the effects of anesthetic agents used on POCD development are still not clear and larger studies should be conducted.

Conclusion. a conclusion, we think that isoflurane and propofol can be used safely in controlled hypotension. isoflurane, despite the lower remifentanil dose, compared to propofol; we recommend that it is preferred as a priority in cases where controlled hypotension will be applied due to lower, more stable blood pressure and heart rate values, faster extubation and early recovery, and higher cerebral oxygen values.

We think that our study is one of the few studies in which propofol and isoflurane are divided into two groups as TIVA and balanced general anesthesia, four different types of surgery are included in otorhinolaryngology surgery, BIS and NIRS monitoring are used together and postoperative cognitive functions are investigated.

Highlights Controlled hypotension is frequently preferred in cases of otolaryngology by reducing bleeding, creating a quality surgical field, increasing operation success, and reducing surgical complications.

- Controlled hypotension can disrupt target organ perfusion, causing various side effects.

- isoflurane and propofol can be used safely in controlled hypotension.

- Our study is one of the few studies in which propofol and isoflurane are divided into two groups as TIVA and balanced general anesthesia, four different types of surgery are included in otorhinolaryngology surgery.

This research did not receive any specific grant from funding agencies in the public, commercial, or notfor-profit sectors. 


\section{References}

1. Degoute CS (2007) Controlled hypotension: A guide to drug choice. Drugs 67: 1053-1076.

2. Varol A, Basa S, Ozturk S (2010) The role of controlled hypotension upon transfusion requirement during maxillary down fracture in double-jaw surgery. J Craniomaxillofac Surg 38: 345-349.

3. Boos GL, Soares LF, Oliveira Filho GR (2005) Postoperative cognitive dysfunction: Prevalence and associated factors. Rev Bras Anestesiol 55: 517-524.

4. Ettinger KS, Yildirim Y, Weingarten TN, Van Ess JM, Viozzi CF, et al. (2016) Hypotensive anesthesia is associated with shortened length of hospital stay following orthognathic surgery. J Oral Maxillofac Surg 74: 130-138.

5. Prasant MC, Kar S, Rastogi S, Hada P, Ali FM, et al. (2014) Comparative study of blood loss, quality of surgical field and duration of surgery in maxillofacial cases with and without hypotensive anesthesia. $\mathrm{J}$ Int Oral Health 6: 18-21.

6. Lin S, McKenna SJ, Yao CF, Chen YR, Chen C (2017) Effects of hypotensive anesthesia on reducing intraoperative blood loss, duration of operation, and quality of surgical field during orthognathic surgery: A systematic review and meta-analysis of randomized controlled trials. J Oral Maxillofac Surg 75: 73-86.

7. Lonjaret L, Lairez O, Manville V, Geeraerts T (2014) Optimal perioperative management of arterial blood pressure. Integr Blood Press Control 7: 49-59.

8. Coşkun D, Celebi H, Karaca G, Karabıyık L (2010) Remifentanil versus fentanyl compared in a targetcontrolled infusion of propofol anesthesia: Quality of anesthesia and recovery profile. J Anesth 24: 373379.

9. Uzun S, Yuce Y, Erden A, Aypar U (2014) Impact of perioperative lidocaine infusion and bis monitorization on remifentanil dosage in hypotensive anesthesia. Eur Rev Med Pharmacol Sci 18: 559565.

10. Yuan X, Liu T, Hu C, Shen X (2019) Comparison of surgical field visibility during propofol or desflurane anesthesia for middle ear microsurgery. BMC Anesthesiol 19: 85.

11. Grundmann U, Silomon M, Bach F, Becker S, Bauer M, et al. (2001) Recovery profile and side effects of remifentanil-based anesthesia with desflurane or propofol for laparoscopic cholecystectomy. Acta Anaesthesiol Scand 45: 320-326.

12. Mahli A, Coskun D, Karaca GI, Akcali DT, Karabiyik L, et al. (2011) Target-controlled infusion of remifentanil with propofol or desflurane under bispectral index guidance: Quality of anesthesia and recovery profile. J Res Med Sci 16: 611-620 .

13. Tavlan A, Topal A, Tuncer S, Kara I, Otelcioglu S (2006) Remifentanil ile Kombine Edilen Propofol ve Desfluran Anestezisinin Hemodinami ve Derlenme Yonunden Karşılaştırılması. Turkiye Klinikleri J Anest Reanim 4: 95-100.

14. Luginbuhl M, Wuthrich S, Petersen-Felix S, Zbinden AM, Schnider TW (2003) Different benefit of bispectal index (BIS) in desflurane and propofol anesthesia. Acta Anaesthesiol Scand 47: 165-173.

15. Triem JG, Rohm KD, Boldt J, Piper SN (2006) Comparison of a propofol- based anesthesia regimen using optimated-target-controlled-infusion (OTCI) and manually-controlled infusion (MCI) technique. Anasthesiol Intensivmed Notfallmed Schmerzther 41: 150-155.

16. Breslin DS, Mirakhur RK, Reid JE, Kyle A (2004) Manual versus target-controlled infusions of propofol. Anaesthesia 59: 1059-1063.

17. Young WL (1992) Effects of desflurane on the central nervous system. Anesth Analg 75: S32-S37.

18. Ruzman T, Simurina T, Gulam D, Ruzman N, Miskulin M (2017) Sevoflurane preserves regional cerebral oxygen saturation better than propofol: Randomized controlled trial. J Clin Anesth 36: 110-117.

19. Ko S-H, Cho YW, Park SH, Jeong JG, Shin SM, et al. (2012) Cerebral oxygenation monitoring of patients during arthroscopic shoulder surgery in the sitting position. Korean J Anesthesiol 63: 297-301.

20. Hayashida M, Chinzei M, Komatsu K, Yamamoto H, Tamai H, et al. (2003) Detection of cerebral hypoperfusion with bispectral index during pediatric cardiac surgery. Br J Anaesth 90: 694-698.

21. Erdem AF, Kayabasoglu G, Tas Tuna A, Palabiyik O, Tomak Y, et al. (2016) Effect of controlled hypotension on regional cerebral oxygen saturation during rhinoplasty: A prospective study. J Clin Monit Comput 30: 655-660.

22. Niazi AA (2016) Postoperative cognitive function and controlled hypotensive anesthesia in patients undergoing septoplasty. Egyptian Journal of Anaesthesia 32: 61-66.

23. Salazar D, Sears BW, Aghdasi B, Only A, Francois A, et al. (2013) Cerebral desaturation events during shoulder arthroscopy in the beach chair position: Patient risk factors and neurocognitive effects. J Shoulder Elbow Surg 22: 1228-1235.

24. Rodriguez IA, Smailagic N, Figuls MR, Ciapponi A, Perez ES, et al. (2015) Mini-Mental State Examination (MMSE) for the detection of Alzheimer's disease and other dementias in people with mild cognitive impairment (MCI). Cochrane Database Syst Rev. 
25. Geng YJ, Wu QH, Zhang RQ (2017) Effect of propofol, sevoflurane, and isoflurane on postoperative cognitive dysfunction following laparoscopic cholecystectomy in elderly patients: A randomized controlled trial. J Clin Anesth 38: 165-171.

26. Singh SK, Kumar A, Mahajan R, Katyal S, Mann S (2013) Comparison of recovery profile for propofol and sevoflurane anesthesia in cases of open cholecystectomy. Anesth Essays Res 7: 386-389. 